\title{
Contextual emergence of mental states
}

\author{
Harald Atmanspacher ${ }^{1}$
}

Received: 24 February 2015/ Accepted: 1 May 2015/Published online: 28 May 2015

(C) Marta Olivetti Belardinelli and Springer-Verlag Berlin Heidelberg 2015

\begin{abstract}
The concept of contextual emergence has been proposed as a non-reductive, yet well-defined relation between different levels of description of physical and other systems. It yields a formally sound and empirically applicable procedure to translate between descriptive levels in an overall consistent fashion. This will be discussed for the contextual emergence of mental states from a neural level of description.
\end{abstract}

Keywords Bridge laws $\cdot$ Coarse graining - Cognitive neuroscience - Emergence - Interlevel relations · Mechanics - Mental causation Philosophy of science . Philosophy of mind · Reduction - Symbolic dynamics . Thermodynamics

\section{Introduction}

A basic strategy for the scientific description of any system, physical or otherwise, is to specify its state and the properties associated with that state and then introduce their evolution in terms of dynamical laws. This strategy presupposes that the boundary of a system can be defined with respect to its environment, although such a definition is often problematic. If it can be achieved, there is usually more than one possibility for specifying states and properties. The fact that states and properties can be formally

This article is part of the Special Issue on 'Complexity in brain and cognition' and has been edited by Cees van Leeuwen.

Harald Atmanspacher

atmanspacher@collegium.ethz.ch

1 Collegium Helveticum, University and ETH Zurich, Zurich, Switzerland and rigorously defined in fundamental physical theories (such as quantum theory) distinguishes the structure of such theories as particularly transparent.

The situation is different in physical theories which are not regarded as fundamental (such as thermodynamics), or in descriptive approaches beyond physics (such as chemistry, biology, psychology, or social sciences). Though states and associated properties are often precisely defined in such theories, these descriptions are typically considered as less rigorous and as lacking the compact structure of fundamental theories. This circumstance provides one (though not the only) motivation for attempts to relate descriptions of systems which are less fundamental to descriptions which are more fundamental.

The sciences know various types of relationships among levels of descriptions of particular phenomena-most common are versions of reduction and of emergence. ${ }^{1}$ Although these domains are not ordered strictly hierarchically, one often speaks of lower and higher levels of description, where lower levels are typically considered as more fundamental. As a rule, phenomena at higher levels of description are regarded as more complex than phenomena at lower levels. This increasing complexity depends on contingent conditions, so-called contexts, that must be taken into account for an appropriate description.

\footnotetext{
${ }^{1}$ Informative discussions of various types of emergence versus reductive interlevel relations are due to Beckermann et al. (1992), Gillett (2002), Butterfield (2011), and Chibbaro et al. (2014). Nevertheless, interlevel relations in general and emergence in particular are often addressed in unspecific and abstract terms that are overly simplistic and confuse matters more than they clarify them. By contrast, contextual emergence is formally precise and empirically applicable across scientific disciplines, from physics and chemistry to neuroscience and cognitive science.
} 
Quite a number of approaches have been proposed to implement interlevel relationships in a system theoretical way, applicable to interdisciplinary topics. Such proposals include center manifold theory (Carr 1981), synergetics (Haken 1983), symbolic dynamics (Lind and Marcus 1995), computational mechanics (Shalizi and Crutchfield 2001), observable representations (Schulman and Gaveau 2005), matrix decomposition (Deuflhard and Weber 2005), and the theory of almost invariant subsets (Froyland 2005). A basic feature of them, expressed in different ways, is the identification of stable state space partitions. Such partitions depend on the chosen context, and their cells essentially represent statistical states giving rise to emergent properties.

In this introductory text of limited length, I will first present the overarching conceptual scheme of contextual emergence in a system theoretical spirit and restrict myself to quotations (rather than detailed discussions) of successful applications for particular scientific issues in physics and physical chemistry. Then I will be a little more specific about the usefulness of contextual emergence in cognitive neuroscience, where it provides a self-consistent way to define mental states from neurodynamics under constraints set at the mental level. Finally, some ramifications for the philosophy of mind and the philosophy of science will be indicated, again with further references for more details.

\section{Contextual emergence: the conceptual scheme}

Alternatives to basically reductive approaches toward the formulation of interlevel relations have attracted increasing interest in contemporary philosophy of science. In recent years, Bishop and Atmanspacher (2006) developed and applied such an alternative, called contextual emergence; for a review, see Atmanspacher and beim Graben (2009). Briefly speaking, this version of emergence is more flexible than plain reduction on the one hand, where a fundamental description is assumed to "fix everything," and, on the other hand, not as arbitrary as a radical emergence where "anything goes."

Contextual emergence was designed on the basis of conceptual philosophy-of-science deliberations (Bishop and Atmanspacher 2006), utilizing lower-level features as necessary but not sufficient conditions for the description of higher-level features. This can be viably combined with the idea of multiple realization, a key issue in the concept of supervenience (Kim 1993), which poses sufficient but not necessary conditions at the lower level.

The basic idea of contextual emergence is to establish a well-defined interlevel relation between a "lower" level $L$ and a "higher" level $H$ of a system. This is done by a two- step procedure that leads in a systematic and formal way (1) from an individual description $L_{i}$ to a statistical description $L_{s}$ and (2) from $L_{s}$ to an individual description $H_{i}$. This scheme can in principle be iterated across any connected set of descriptions, so that it is applicable to any situation which can be formulated precisely enough to be a sensible subject of scientific investigation.

The essential goal of step (1) is the identification of equivalence classes of individual states that are indistinguishable with respect to a particular ensemble property. This step implements the multiple realizability of statistical states in $L_{s}$ by individual states in $L_{i}$. The equivalence classes at $L$ can be regarded as cells of a partition. Each cell is the support of a (probability) distribution representing a statistical state, encoding limited knowledge about individual states.

The essential goal of step (2) is the assignment of individual states at level $H$ to statistical states at level $L$. This is impossible without additional information about the desired level $H$ description. In other words, it requires the choice of a context setting the framework for the set of observables (properties) at level $H$ that is to be constructed from level $L$. The chosen context provides constraints that can be implemented as stability criteria at level $L$. It is crucial that such stability conditions cannot be specified without knowledge about the context at level $H$. In this sense, the context yields a top-down constraint, or downward confinement (sometimes misleadingly called downward causation).

The notion of stability induced by context is of paramount significance for contextual emergence. Roughly speaking, stability refers to the fact that some system is robust under (small) perturbations. For example, (small) perturbations of a homeostatic or equilibrium state are damped out by the dynamics, and the initial state will be asymptotically retained. The more complicated notion of a stable partition of a state space is based on the idea of coarse-grained states, i.e., cells of a partition whose boundaries are (approximately) maintained under the dynamics.

Such stability criteria guarantee that the statistical states of $L_{s}$ are based on a robust partition so that the emergent observables in $H_{i}$ are well defined. Implementing a contingent context at level $H$ as a stability criterion at $L$ yields a proper partitioning for $L_{s}$. In this way, the lower-level state space is endowed with a new, contextual topology. From a slightly different perspective, the context selected at level $H$ decides which details in $L_{i}$ are relevant and which are irrelevant for individual states in $H_{i}$. Differences among all those individual states at $L_{i}$ that fall into the same equivalence class at $L_{S}$ are irrelevant for the chosen context. In this sense, the stability condition determining the contextual partition at $L_{s}$ is also a relevance condition. 
The interplay of context and stability across levels of description is the core of contextual emergence. Its proper implementation requires an appropriate definition of individual and statistical states at these levels. This means in particular that it would not be possible to construct emergent observables in $H_{i}$ from $L_{i}$ directly, without the intermediate step to $L_{s}$. And it would be equally impossible to construct these emergent observables without the downward confinement arising from higher-level contextual constraints.

In this spirit, bottom-up and top-down strategies are interlocked with one another in such a way that the construction of contextually emergent observables is selfconsistent. Higher-level contexts are required to implement lower-level stability conditions leading to proper lowerlevel partitions, which in turn are needed to define those lower-level statistical states that are co-extensional (not necessarily identical!) with higher-level individual states and associated observables.

The procedure of contextual emergence has been shown to be applicable to a number of examples from the sciences. Paradigmatic case studies are as follows: (1) the emergence of thermodynamic observables such as temperature from a mechanical description (Bishop and Atmanspacher 2006), (2) the emergence of hydrodynamic features such as Rayleigh-Bénard convection from manyparticle theory (Bishop 2008), and (3) the emergence of molecular observables such as chirality from a quantum mechanical description (Primas 1998; Bishop and Atmanspacher 2006).

If descriptions at $L$ and $H$ are well established, as it is the case in these examples, formally precise interlevel relations can be straightforwardly set up. The situation becomes more challenging, though, when no such established descriptions are available, e.g., in cognitive neuroscience or consciousness studies, where relations between neural and mental descriptions are considered. Even there, contextual emergence has been proven viable for the construction of emergent mental states (Atmanspacher and beim Graben 2007; Allefeld et al. 2009). The following section sketches the basic elements of this procedure and illustrates it by a concrete example.

\section{Contextual emergence of mental states and observables}

A basic way in which systems at any level are described starts with the specification of their states, their observables, and their dynamics. An appropriate representation of these basic elements is usually given in terms of a state space. The state of a system is represented by a more or less refined subset of that space, the values of its associated observables are projections of that subset onto the state space coordinates, and the dynamics is represented by the motion of the state as parametrized by time.

Let us assume a (lower level) neural state space $X$ with fine-grained states $x$, ideally represented pointwise in $X$, and with observables $X_{i}, i=1, \ldots, n$, for $n$ degrees of freedom. Typical examples for neural observables are electroencephalogram (EEG) potentials at the macroscopic level, local field potentials at the mesoscopic level, or spike trains of neurons at the microscopic level of brain activity. These observables are usually obtained with much higher resolution than observables at a mental level of description. There is agreement that neural correlates of mental states are to be found at the level of macroscopic or mesoscopic brain dynamics, but hardly at the level of individual neurons. ${ }^{2}$

The construction of a (higher level) mental state space $Y$ from $X$ can be based on some coarse graining of $X$. That is, the state space $X$ must be partitioned such that cells of finite volume in $X$ emerge, which can be used to represent mental states in $Y$. Often, such discrete states are denoted by symbols $A, B, C, \ldots$, where each symbol represents an equivalence class of neural states. In contrast to the (quasi)continuous dynamics of states $x$ in a state space $X$, the dynamics in $Y$ is a discrete sequence of symbols as a function of time, briefly a symbolic dynamics.

A coarse-grained partition on $X$ implies neighborhood relations between mental states in $Y$ that are different from those in the original state space $X$; in this sense, it implies a change in topology. Also, the definition of observables $Y_{i}$ for $Y$ implies an algebra of mental observables that is different from that of neural observables. Obviously, these two differences depend essentially on the choice of the partition of $X$.

First of all, it should be required that a proper partition leads to mental states in $Y$ that are empirically plausible. For instance, one very fundamental plausible formation of equivalence classes of neural states is due to the distinction between wakefulness and sleep-two evidently different mental states. ${ }^{3}$ However, an important second demand is that these equivalence classes be stable under the dynamics in $X$, which emphasizes the importance of the dynamical domain for contextual emergence. If this stability cannot be

\footnotetext{
${ }^{2}$ It is possible that global brain activity is too coarse for subtle distinctions between fine-grained mental states. In this case, dynamics at the mesoscopic level may still be viable candidate.

3 An empirically based relation between mental state space representations of wakefulness versus sleep and their corresponding neural state space representations (and other, subtler examples, such as selective attention, intrinsic perceptual selection) has been reported by Fell (2004). For alternative state space approaches, see Wackermann (1999) or Hobson et al. (2000). These studies do, however, not utilize contextual emergence, but rather look for neural correlates of mental states in the usual, conventional sense, which does not ask for any theoretical understanding of those correlations.
} 
guaranteed, the boundaries between cells in $Y$ become blurred as time proceeds, thus rendering the concept of a mental state ill-defined.

The required stability criterion depends on contexts to be set at the higher (mental) level. For empirical applications, this is most effectively done by a properly defined experimental paradigm. For instance, an experiment in which two different cognitive tasks are to be distinguished sets the context for a bipartition of states in the lower (neural) state space. How this bipartition looks exactly is determined by the detailed data analytical procedure indicated below. Other (broader) contexts, inducing other partitions, can be socially or culturally relevant boundary conditions of an investigation.

From a theoretical point of view, Chalmers (2000) suggested "phenomenal families" as sets of mutually exclusive phenomenal (mental) states. These families are hierarchically structured, e.g., cognitive states, can be desires, beliefs, perceptions etc., perceptions can be visual or else, visual perception can be about color or else, color perception can be about blue or else, etc. The more finegrained the distinctions are, the more difficulty will be expected for a proper identification of equivalence classes of corresponding neural states.

Beim Graben and Atmanspacher (2006) and Atmanspacher and beim Graben (2007) showed in detail that a particular type of partition is needed for a proper definition of stable states in $Y$ based on cells in $X$. These partitions are called Markov partitions, ${ }^{4}$ tightly related to the class of generating partitions. Such partitions can be identified by the fact that their dynamical entropy is the supremum over all possible partitions, the so-called Kolmogorov-Sinai entropy (see Atmanspacher (1997) for an annotated introduction).

A pertinent example for the successful application of contextual emergence to experimental data was worked out by Allefeld et al. (2009), using EEG data from subjects with sporadic epileptic seizures. This means that the neural level is characterized by brain states recorded via EEG. The context of normal versus epileptic mental states essentially requires a bipartition of that neural state space.

The data analytic procedure rests on ideas by Gaveau and Schulman and Gaveau (2005), Froyland (2005), and Deuflhard and Weber (2005). It starts with a 20-channel EEG recording, giving rise to a state space of dimension 20 , which can be reduced to a lower number by restricting to principal components (PC). On the resulting low-dimensional state space, a homogeneous grid of cells is imposed in order to set up a Markov transition matrix $T$

\footnotetext{
${ }^{4}$ Markov partitions minimize correlations between their cells, thus creating a Markov process for the symbolic dynamics in $Y$ if the dynamics in $X$ is chaotic. Evidence for chaotic brain processes has often been reported (cf. Kaneko and Tsuda 2000, and references therein).
}

reflecting the EEG dynamics on a fine-grained auxiliary partition (which is not yet the desired bipartition).

The eigenvalues of $T$ express relaxation timescales for the dynamics which can be ordered by size. Gaps between successive relaxation times indicate groupings referring to mental states defined by neural state partitions of increasing refinement. The first group is often sufficient for the distinction of "target" mental states. But, in a hierarchical situation, one may even be able to find substates within those "target" states whose relaxation times form a second-order group, and so on. This has been demonstrated in numerical simulations of "nested" dynamical systems such as pairs of multiple fixed point attractors with different coupling strengths (Allefeld et al. 2009, Sect. IV).

The eigenvectors corresponding to the eigenvalues span an eigenvector space, in which the measured PC-compactified states form a simplex. For instance, three leading eigenvalues allow a representation of neural states in a twodimensional eigenvector space which yields a two-simplex with three vertices (a triangle). Classifying the measured neural states according to their distance from the vertices of the simplex then leads to three clusters of neural data. They can be coded and identified in the PC state space (Allefeld and Bialonski 2007), where the clusters appear as nonintersecting convex sets distinguishing one normal state and one seizure state (composed of two substates). For details, see Allefeld et al. (2009, Sect. V).

Finally, the result of the partitioning can be inspected in the originally recorded time series to check whether mental states are reliably assigned to the correct episodes in the EEG dynamics. The study by Allefeld et al. (2009) shows perfect agreement between the distinction of normal and epileptic states and the bipartition resulting from the spectral analysis of the neural transition matrix. (For more technical details, which are beyond this introductory paper, readers are encouraged to consult the quoted literature).

\section{Other applications and perspectives}

An important feature in contextual emergence is the topological equivalence of representations in neural and mental state spaces (cf. Metzinger 2003, p. 619, and Fell (2004) for empirically based examples). Topological equivalence ensures that the mapping between $X$ and $Y$ is faithful in the sense that the two state space representations yield compatible information about the system. ${ }^{5}$ Non-

\footnotetext{
5 Two representations of dynamical systems $M=(X, \phi)$ and $N=$ $(Y, \psi)$ with flows $\phi, \psi$ in state spaces $X, Y$ are topologically equivalent if the mapping $\pi: M \mapsto N$ is continuous and invertible, and its inverse $\pi^{-1}$ is also continuous. In this case, the flows are related by $\phi=\pi^{-1} \psi \pi$.
} 
generating partitions provide incompatible representations in $Y$ that are topologically inequivalent with the underlying representation in $X$. Conversely, compatible mental models that are topologically equivalent with their neural basis emerge if they are constructed from generating partitions.

In this framework, contextual emergence was recently shown to contribute to a better understanding of a longstanding puzzle in the philosophy of mind: mental causation. It dissolves the alleged conflict between the horizontal (intralevel) and vertical (interlevel) determination of mental events as ill-conceived (Harbecke and Atmanspacher 2011). The key point is a construction of a properly defined mental dynamics topologically equivalent with the dynamics of underlying neural states. The statistical neural states based on a proper partition are then coextensive (but not identical) with individual mental states.

As a consequence, (1) mental states can indeed be causally and horizontally related to other mental states, and (2) they are causally related neither to their vertical neural determiners nor to the neural determiners of their horizontal effects. ${ }^{6}$ This makes a strong case against a conflict between a horizontal and a vertical determination of mental events and resolves the problem of mental causation in a deflationary manner. Vertical determination and horizontal determination do not compete, but complement one another in a cooperative fashion.

Other recent work applying contextual emergence in the philosophy of mind refers to the debate about the "intentional stance" according to Dennett (1989). Beim Graben (2014) reconstructed a hierarchy of necessary and sufficient conditions for the applicability of the intentional strategy à la Dennett based on the scheme of contextual emergence. In this manner, intentionality can be considered as a contextually emergent property at many hierarchical levels.

A recent contribution by Atmanspacher et al. (2014) with respect to the issue of reproducibility as a methodological pillar of science utilized the relevance condition of contextual emergence to deal with the problem of identifying relevant observables as the target to be reproduced. This is a vexing problem in interdisciplinary research in general and translational biomedicine in particular. A corresponding "relation of relevance" (Van Fraassen 1980) between the complexity of a system and the granularity of its description can be operationally implemented by contextual emergence.

Finally, it should be emphasized that contextual emergence was primarily conceived as a relation between levels of descriptions, not levels of nature: It addresses questions

\footnotetext{
${ }^{6}$ The notion of causation as it is used here, and in the literature on mental causation, refers to causes that temporally precede their effects. This makes no sense in a synchronously conceived vertical interlevel relation.
}

of epistemology rather than ontology. However, it would be desirable to know whether and how this can be transferred to ontological interlevel relations. A possible option to relate epistemic and ontic stances along the lines of Quine's (1969) ontological relativity was indicated by Atmanspacher and Kronz (1999). This challenges the old idea of one fundamental ontology from which everything else derives. At the same time, it is specific enough to resist the backlash into a relativist patchwork of unconnected model fragments.

\section{References}

Allefeld C, Atmanspacher H, Wackermann J (2009) Mental states as macrostates emerging from EEG dynamics. Chaos 19:015102

Allefeld C, Bialonski S (2007) Detecting synchronization clusters in multivariate time series via coarse-graining of Markov chains. Phys Rev E 76:066207

Atmanspacher H (1997) Dynamical entropy in dynamical systems. In: Atmanspacher H, Ruhnau E (eds) Time, temporality, now. Springer, Berlin, pp 327-346

Atmanspacher H, Bezzola Lambert L, Folkers G, Schubiger PA (2014) Relevance relations for the concept of reproducibility. J R Soc Interface 11(94):20131030

Atmanspacher H, beim Graben P (2009) Contextual emergence. Scholarpedia 4(3):7997. http://www.scholarpedia.org/article/Co ntextual_emergence

Atmanspacher H, beim Graben P (2007) Contextual emergence of mental states from neurodynamics. Chaos Complex Lett 2:151-168

Atmanspacher H, Kronz F (1999) Relative onticity. In: Atmanspacher H, Amann A, Müller-Herold U (eds) On quanta, mind and matter: Hans Primas in context. Kluwer, Dordrecht, pp 273-294

Beckermann A, Flohr H, Kim J (1992) Emergence or reduction? de Gruyter, Berlin

beim Graben P (2014) Contextual emergence of intentionality. J Conscious Stud 21(5/6):75-96

beim Graben P, Atmanspacher H (2006) Complementarity in classical dynamical systems. Found Phys 36:291-306

Bishop RC (2008) Downward causation in fluid convection. Synthese 160:229-248

Bishop RC, Atmanspacher H (2006) Contextual emergence in the description of properties. Found Phys 36:1753-1777

Butterfield J (2011) Emergence, reduction and supervenience: a varied landscape. Found Phys 41:920-960

Carr J (1981) Applications of centre manifold theory. Springer, Berlin

Chalmers D (2000) What is a neural correlate of consciousness? In: Metzinger T (ed) Neural correlates of consciousness. MIT Press, Cambridge, pp 17-39

Chibbaro S, Rondoni L, Vulpiani A (2014) Reductionism, emergence, and levels of reality. Springer, Berlin

Dennett D (1989) The intentional stance. MIT Press, Cambridge

Deuflhard P, Weber M (2005) Robust Perron cluster analysis in conformation dynamics. Linear Algebra Appl 398:161-184

Fell J (2004) Identifying neural correlates of consciousness: the state space approach. Conscious Cogn 13:709-729

Froyland G (2005) Statistically optimal almost-invariant sets. Phys D 200:205-219

Gillett C (2002) The varieties of emergence: their purposes, obligations and importance. Grazer Philosophische Studien 65:95-121 
Haken H (1983) Synergetics. Springer, Berlin

Harbecke J, Atmanspacher H (2011) Horizontal and vertical determination of mental and neural states. $\mathrm{J}$ Theor Philos Psychol 32:161-179

Hobson JA, Pace-Schott EF, Stickgold R (2000) Dreaming and the brain: toward a cognitive neuroscience of conscious states. Behav Brain Sci 23:793-842

Kaneko K, Tsuda I (2000) Complex systems: chaos and beyond. Springer, Berlin

Kim J (1993) Supervenience and mind. Cambridge University Press, Cambridge

Lind D, Marcus B (1995) Symbolic dynamics and coding. Cambridge University Press, Cambridge

Metzinger T (2003) Being no one. MIT Press, Cambridge

Primas H (1998) Emergence in exact natural sciences. Acta Polytech Scand 91:83-98
Quine WV (1969) Ontological relativity. In: Quine WV (ed) Ontological relativity and other essays. Columbia University Press, New York, pp 26-68

Schulman LS, Gaveau B (2005) Dynamical distance: coarse grains, pattern recognition, and network analysis. Bulletin des Sciences Mathematiques 129:631-642

Shalizi CR, Crutchfield JP (2001) Computational mechanics: pattern and prediction, structure and simplicity. J Stat Phys 104:819-881

van Fraassen B (1980) The scientific image. Clarendon, Oxford

Wackermann J (1999) Towards a quantitative characterisation of functional states of the brain: from the non-linear methodology to the global linear description. Int J Psychophysiol 34:65-80 